\title{
A Propulsive Roadmap for IoT Beyond 2025
}

\author{
Manas Kumar Yogi \\ Asst. Prof. Department of Computer Science, Pragati Engineering College(A), Surampalem,A.P. \\ Email: manas.yogi@gmail.com \\ K. Ganga Devi Bhavani \\ M.Tech. Student, Department of Computer Science, Pragati Engineering College(A), Surampalem,A.P. \\ Email: devikakarla258@gmail.com
} \begin{abstract}
-
IoT is a worldwide-recognized trend that is gaining popularity incredibly fast. The baseline lies in the fact that IoT has already transformed a number of industries and took them to the new level, and these industries include healthcare, finances and much more. No wonder there is such a hype about it There are tremendous new opportunities with IoT flowing out every couple of months so we highly recommend all to keep an eye on this technology. In this paper will have shed light on the inherent concepts which will affect the working of IoT beyond 2025. We have discussed key points in this paper regarding the operational components of an IoT System where improvements can be done by researchers so as to leverage the usage of IoT environment.
\end{abstract}

Keywords - IoT, Nano, Sensors, Cognitive, network, RFID.

Date of Submission: July 24, 2018

Date of Acceptance: Aug 22, 2018

\section{INTRODUCTION}

The Internet of Things may be a captivating issue in the business anyway it is definitely not another thought. In the mid 2000's, Kevin Ashton was laying the arrangement for what may transform into the Internet of Things (IoT) at MIT's AutoID lab. Ashton was one of the pioneers who envisioned this idea as he examined for ways that Delegate and Gamble could improve its business by interfacing RFID information to the Internet. The thought was essential yet competent. If all things in step by step life were furnished with identifiers and remote accessibility, these things could be talk with each other and be regulated by PCs.

The term Internet of Things (IOT) has been around for a long time. In this circumstance, it is gaining ground with the improvement of front line remote development. The principal thought of this thought is the closeness of an arrangement of articles -, for instance, RFID, NFC, sensors, actuators, mobile phones. In this IOT development the RFID is the most basic thought and it is essential for web of things. Unmistakable developments in publicize like RFID, machine to machine correspondence, vehicle to vehicle correspondence molecule etc. are actualized using IOT[8]. The basic issue of IOT is going up against circumstance of security the potential Hackers who always on edge to strike. The ability to code and track objects has empowered associations to end up more powerful, quicken shapes, lessen bumble, keep away from robbery, and join mind boggling and adaptable legitimate systems through IOT.

The "Internet of Things" alludes to the coding and systems administration of ordinary items and things to render them independently machine - clear and traceable on the Internet Much existing substance in the Internet of Things has been made through coded RFID labels.

\section{Identification technology-thing DNA identifier}

Over the significant lot, the Internet of Things might be viable in case we make sense of how to relate all the different devices in an especially uncomplicated way. At the present time, the nonappearance of interoperability is baffling expansive utilize. An average tongue could be an answer and the contraptions itself need to wind up more watchful. A characteristic living being passes on in each cell the aggregate genotype with solitary "working rules". This could be a perspective for the Internet of Things.

Today, all the different devices with their individual limits inside the Internet of Things give by methods for their own, prohibitive procedures. Honestly, there are presently an extensive variety of genuine standards, it's essentially that these restrictive occur inside each individual industry. If you have to relate the particular regions and devices with each other, a foundation quickly ends up being astoundingly awesome.

A focal control unit makes IoT environments susceptible: The normal "connector" to date is by methods for a principle issue. Sensors assemble data and send these to a gateway or cloud server. Starting there the data is deciphered and actuators controlled suitably. In the field of building robotization, this can frequently be things like warming controls, light switches, modernized entryway or shade controls[9]. In solitude nevertheless, without the central section or cloud game plan, most of the sensors and actuators are all around defenseless. Just by virtue of learning inside that central control unit they can fulfill their work and team up with each other.

\section{INTERNET OF THINGS ARCHITECTURE AND TECHNOLOGY}

\section{A. Cognitive Architectures:}

\section{Cognitive computing}

Cognitive computing mimics human senses. One connection between big data analysis and cognitive computing is human's big data thinking. The experience constantly accumulates during the life of human being. The second level is pursuing spiritual culture and the third level is concerned with the meaning of life. The amount of people in top level is the least. Currently, the thinking that is simulated by machine intelligence mainly focuses on 
first and second level to concern the living standard and the emotional state of human. The corresponding applications are: health monitoring, smart healthcare, smart home, smart city and emotional care. The third level is further deep that concerns the meaning of life and puts forward personalized suggestions for development direction in the life of user to help the user realize happy but more meaningful life. The enabling technologies of cognitive computing include reinforcement learning and deep learning. The reinforcement learning can learn from the environment and reflects on behavior. The deep learning can learn high levels of features[10].

\section{i. Cognitive Computing And Reinforcement Learning}

The conventional machine learning techniques can partitioned into directed learning and unsupervised learning. In those strategies, the machines prepare those model with information that are frequently in settled organization and machines finish undertakings, for example, relapse, grouping and accumulation. In any case, the data that can be gotten by the machines is restricted. It is troublesome for machines to learn data in nonlinear case since they can just lead expectation as per the got data. Besides, the tag for same information can be distinctive in various conditions, which implies that the ease of use of data learned by machines is diverse for various clients. Customary managed learning and unsupervised learning depend on shut preparing with information input. These customary learning strategies can't meet the necessities of reasonable change in knowledge of machine. Thusly, fortification learning has turned into a hot research branch in the field of machine learning.

Support learning is very like the learning procedure of human. How about we take the situation where a youngster figures out how to talk for instance. At the point when a youngster is to take in a word, as a rule a grown-up would over and again read that word, pointing at something spoke to by that word or doing the activity spoke to by that word. On the off chance that the kid's understanding isn't right because of mistaken judgment, the grown-up would lead revision. Once the tyke takes care of business, the grown-up would give remunerate. The encompassing condition is additionally a critical factor amid the learning procedure of human. Support learning takes the case by this point and it can gain from nature and think about conduct. An arrangement of reward instruments are set up, i.e., certain reward is given when some conduct is useful for objective and certain discipline is applied actually. Amid the procedure toward the target, there are numerous decisions. In this way, the choice at each time isn't really the ideal, yet it must be useful for the machine to acquire remunerate.

Information are created during the time spent endeavors of machine, the last target isn't relapse, characterization or conglomeration yet most extreme reward. With this reason, both effective and unsuccessful endeavors are significant for the machine. It would gain as a matter of fact in past endeavors at each resulting venture. Be that as it may, if a machine just speaks with itself, at that point its discernment isn't agreeable. This is much the same as for a talk, it is hard to figure out how to talk without speaking with others. Along these lines, if a learning framework completes its thought regardless of outer conditions, it's anything but a decent cognitive framework[11]. In this way, a cognitive framework should lead correspondence straightforwardly with human. Be that as it may, if a man is extraordinarily allocated to lead correspondence with a machine, it would expend a great deal of time and labor. Luckily, the group sourcing strategy can make the correspondence amongst machine and human common.

\section{ii. Cognitive Computing And Image Understanding}

If machine is going to solve problems in real world, the best way is to simulate the thinking mode of human brain. In cognitive system, the features can either be extracted from original data for classification and prediction model using the method of manual feature design to simulate the logical thinking ability of human brain or can be learned through deep learning to simulate the ability of visual thinking of human brain.

The concept of IoT includes three meanings. The first is that its core and basis are the Internet and it is a kind of extension of the Internet. The second is that the terminals in IoT are applied to any things that are connected each other by wire or wireless communication through RFID, GPS, laser scanner, infrared sensor and other sensors. The third is that there is a business process flow corresponding to a given application. Besides the three meanings stated above, the concept of CIoT is assigned a new implication namely the intelligence. Cognition can impenetrate any parts of IoT and acts as an important role for a given application[4]. The figure 1 illustrates CIoT's sketch map of topology.

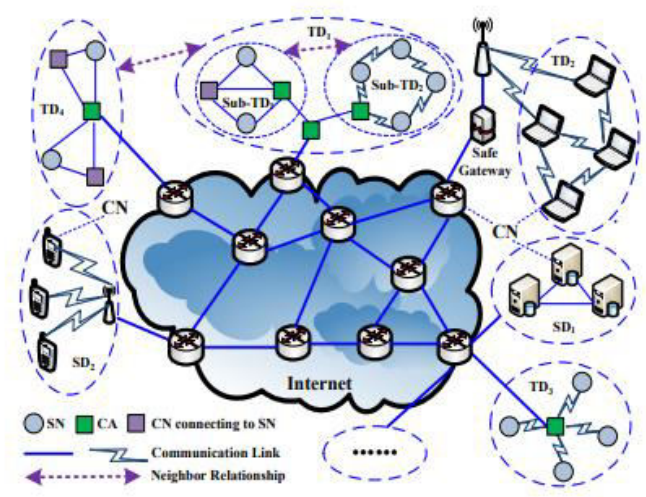

Figure 1: Cognitive Internet of Things Architecture Things Domain: CIoT are grouped into Things Domains (TDs), which are defined as $\mathrm{TD}_{1}, \mathrm{TD}_{2}, \mathrm{TD}_{3} \ldots \mathrm{TD}_{\mathrm{n}}$. TDs are high coupled and relatively independent. Each thing with RFID, GPS and various sensors can be regarded as a node in TD. Generally, for an application of CIoT, there is at least one TD. Sometimes TD can be divided into several Sub-TDs. For example, we can think of the things of a plant as a TD, thus, the things of each workshop can be thought as a Sub-TD. The nodes could be divided into two 
types: Cognitive Nodes (CNs) and Simple Nodes (SNs) according to the capability of nodes[12].

Definition 1: $\mathrm{CN}$ refers to the node which has the ability to autonomously perform intelligent actions according to acquired information and prior knowledge, such as perception information merging, routing, and business process decision making. CN possesses higher process capability than $\mathrm{SN}$. It acts as decision-making role and establishes the communication between any parts of CIoT. In generally, CNs includes computers, servers, routers and other intelligent devices.

Definition 2: SN refers to the node without intelligence and having less process capability than $\mathrm{CN}$. The concept of $\mathrm{SN}$ is opposite to the $\mathrm{CN}$. There are different numbers of CNs in different TDs and maybe only one $\mathrm{CN}$ in a TD under special circumstances. If a TD includes multiple $\mathrm{CNs}$, two or more CNs can cooperate to enhance capability according to requirements[13]. Furthermore, two or more TD's can also cooperate if necessary.

Definition 3: For an application orienting far broad network environment, the cooperation should relate to two or more $\mathrm{CNs} / \mathrm{TDs}$, which is called Multi-x Cooperation.

Definition 4: For Multi-x Cooperation, the specific CNs selected to carry out cooperative assignments in a TD are called Cognitive Agents (CAs).

Definition 5: Two TDs with directly cooperative relationship are reciprocally called neighbors, and two TDs with indirectly cooperative relationship in virtue of other TDs are reciprocally called extended neighbors. For example, both TD1 and TD4 and Sub-TD1 and Sub-TD2 can be called neighbors.

\section{B. Experiential Architectures:}

This architecture can be described from bottom to top in the figure. It is developed for a clear outline of practical or experiential applications in IOT.

Layer 1: The base and center piece of IOT application is the sensors and electronic gadgets that can associate with things and snatch the information from it.

Layer 2: Sensors gather the information that have to change over it into justifiable configuration and interface those sensor gadgets utilizing some convention.

Layer 3: Network connectivity; connect your device with wireless connectivity or internet wired connection. This connectivity is changed based on context \& domain.

Layer 4: Data gathering is done and various ways to store data is discussed here.
Layer 5: This layer is security layer or application abstraction layer or data abstraction where we can apply security to our product.

Layer 6: It is presentation layer or decision taken layer. Based on the requirement we can display reports or applying machine learning or some custom logic and takes smart decision and send signal back to the sensors.

Layer 7: User will interact where our actually product and business logic comes into picture.

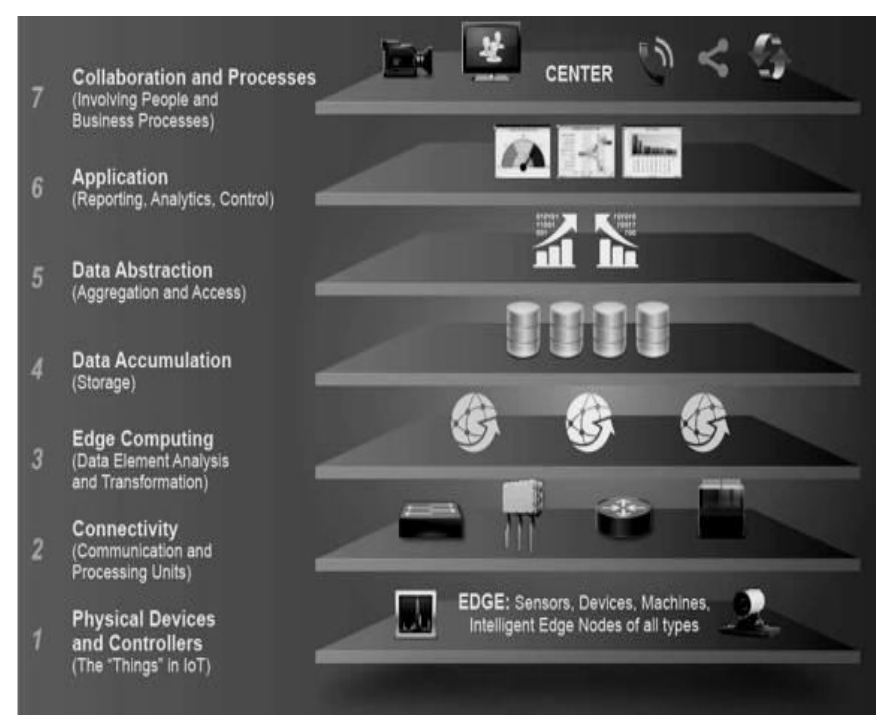

Figure 2: Experiential Architectures

\section{Communication TeChNOLOGY}

\section{a. Unified protocol over wide spectrum:}

Currently, dissimilar to your IEEE models, there is no unified standard representing the IoT. Each organization that is taking a shot at IoT has shaped their own particular partnership with different organizations and set standard (protocols) with which they work. Various communication technologies are used in the processing IOT applications. Yet there are few majorly used communication technologies like, ZigBee, RF Links, Bluetooth, ZWave[7].

- ZigBee is used over defined area of network for the reason of low data rate. As it takes low power, communication protocol used comes under the Wireless Personal Area Network (WPAN).

- RF Links bounded area is within $100 \mathrm{~m}$ to $1 \mathrm{~km}$. The Radio Frequency Identification technology used to track the objects in the IOT applications, as it is low of cost and mobility features.

- Bluetooth which is a cheap radio technology that connects the neighboring devices which is an advantageous feature in IOT applications to interconnect devices. 
- Z-Wave having open communication protocols which is used in automation and reliable message passing over the network.

\section{b. Network Technology:}

A network is group of devices connected with one another in predefined area to perform some particular task. Here in the IOT Network technology, topology is most important term to work with the IOT devices. In the networking terminology, the term topology described as "how devices are interconnected with one another and organized in particular area of network". The most efficient and popularly used network topologies in IOT applications are Star and Mesh topology.

\section{i. Network cognition :}

Cognitive Radio is a promising empowering agent correspondence innovation for IoT. Its shrewd correspondence worldview is suited to imparting objects having occasion driven nature, which create explosive activity. Cognitive Radio can help beat the issues of impact and over the top conflict in the remote access organize that will emerge because of the arrangement of a few items associated with foundation through radio connections.

Cognitive Radio (CR) has risen as a keen innovation to address the range shortage issues. CR means to utilize the vacant range band when it isn't utilized by the authorized client. A broad research has been done since the commencement of this innovation in 1999 where diverse difficulties like range detecting, participation among CR clients and relevance of CR systems have been generally investigated. In this paper, we give new uses of CR innovation for Internet of Things (IoT) and propose fitting answers for the genuine difficulties in CR innovation that will make IoT more reasonable and appropriate.

\section{ii. Self-learning, self-repairing networks:}

As IOT is based on the concept of ubiquitous computing, with the existing objects like sensors, actuators and mobile devices interact with each other though radio frequency technology that uses unique addresses to communicate with the objects.

Though IOT are designed to achieve a common goal or to perform certain task connected with the predefined network, there are some certain situations where IOT devices need to take their own self decision for the sake of task and also requires self-protection from the intruders. So here comes the concept of self-learning and selfrepairing networks. Based on different criteria, the task that need to be performed by the IOT applications using the IOT devices need to self-organize the network in which the way they need to work. And also to protect from the intruders, IOT devices should self-protect from the intruders.

For this reason, the concept of intrusion detection and prevention introduced in IOT which leads to selfmanaging network of system which should hold the four characteristics: Self configuration, Self-optimization, Selfhealing, Self-protecting.
Self-configuration: The self-configuration property is found in systems that are capable to self-install and self-set to achieve the user goals.

Self-optimization: The self-optimization property is found in systems that can make some changes proactively to improve the performance of the system.

Self-healing: The self-healing property is found in systems that detect and diagnose problems. It is important that the self-healing systems have fault tolerance.

Self-protecting: The self-protecting property is found in systems that protect themselves from malicious attacks. The autonomic system adjusts itself to offer security, privacy and data protection.

\section{SOFTWARE AND ALGORITHMS}

The Internet of Things is turning into something of a nebulous concept, as more and more devices enjoy connected status it's become more difficult to draw a line in the sand between what does and does not constitute as an IoT device. Smart homes and connected cars can include so many different IoT devices it's easy to see that this multi-faceted market shows no sign of slowing, and development continues marching towards progress.

Initially one of the main driving forces behind this IoT revolution was the open source community whose constant experimentation, combined with accelerating technological possibilities, created many new and interesting applications. These applications range from Wi-Fi kettles to smart data analyzing machines and everything in between.

\section{a. User oriented software:}

User oriented software also called as User centered design (UCD) or User Driven Development (UDD). User oriented software is a groundwork of processes where goals like Usability, platform, theme of work of the product or the software should rapidly grow. After that one should make user attract to the software to increase the usability of the product. The question here is "how to increase the usability of the product?" Here comes the idea of Open Source Software, where source code is available one can use it, modify it and even can share it with others.

To increase the growth of IOT, in the categorization of user driven/oriented software various open source software tools. Supporting IOT applications are used at minimum requirement. From mini IOT application to day to day application also characterized using open source software IOT tools. Here, some of the popularly used open source software IOT tools are mentioned below[6].

- DeviceHive - used to control the network by remote disregarding configuration of the network with the help of cloud based API.

- Kaa- used to provide backing between peer to peer systems connected over cloud. 
- Aurdino- comes under both hardware and software which uses Integrated Development Environment to send messages.

- Home Assistant - used for home automation controlled using mobiles or desktop browsers achieved using python.

- Device Hub - provides 'Platform as a service' both hardware and software designed particularly for the IOT projects.

\section{b. The Invisible IoT:}

As the amount of data that is provided by IOT devices need to be saved for the further reference, this will become the issue that need to be faced by organizations. For this reason, various technologies like cloud, bigdata, artificial intelligence and machine learning came into the picture.

The invisible IOT is achieved when IOT devices are connected over a network providing internet and making the devices take autonomous decision without any command provided by the user. The autonomous decision taken by the IOT device based on the task required to be performed or application specific.

\section{c. Easy-to-deploy IoT:}

For the easy deployment of IoT successfully, there are certain guidelines that need to be followed, they are:

- Proper planning like blueprint of the network of devices like blueprint that need to be connected with one other should be prepared

- Broad view of end point devices connected in the network and their behavior.

- Security of the devices in the network and the data they are sharing with.

- Hardware and software requirements of the IoT applications.

\section{d. To-Humans collaboration:}

Today IoT is influencing our everyday communication with "things" around us, and even opens the entryway of potential outcomes for a more economical workplace. Later on, we can anticipate that IoT will create altogether new employment parts and titles and to totally change the way we drive, interact and collaborate.

As the advancement in IoT, artificial intelligence and machine learning contribute more towards the IoT applications. This helps to create an autonomous network that coordinates and collaborates with the other devices in the network. The heterogeneity of network that created establishes the devices collaborate with one other.

Autonomous decision or automation and Interaction collaborate to human factors like privacy, security and control in self- monitoring IoT applications.

\section{e. IoT for All:}

Invoking limited Knowledge in IoT, limited network connectivity and infrastructure one can achieve the out of the box solution to a problem. The way resources are been utilized defines solution to task.
Open source platforms both hardware and software for IoT application are made available. These days the IoT devices hardware infrastructures are available for low price. These IoT applications collaborate with different sectors in IT and business sectors based on the application they are designed for. Starting with small IoT applications to large IoT applications, one can work based on their own standards.

IoT applications can be developed in various sectors like manufacturing, agriculture, construction and various other environments. Information from sensors can enable groups to track the area, temperature or state of a business' advantages and do considerably more with data. Data can be collected and analyzed from millions of sources with IoT. The opportunities for IoT are endless.

\section{f. Self-generating "molecular" software :}

The term "nanomachine" is not clearly defined. It both denotes molecular machines, i.e. nano-scale devices performing some mechanical action, and nanobots, i.e. more complex devices that typically also include a nanosensors and some form of logic or simple computation. Molecular motors are one example for these molecular machines as they are able to move autonomously. Different molecular motors have been developed during the past years, based on bubble, selfelectrophoretic, electrical, magnetic or ultrasound propulsion. Applications are seen in medicine (for example for drug delivery or medical diagnostics) and in environmental monitoring or wastewater treatment. The creation of nanorobots requires the integration of nanosensors, molecular machines with some kind of logic. However, due to missing molecular machines and nanoelectronics and problems to assemble these different parts, nanorobots still require fundamental research.

IoT devices are not stationary any more or are moved passively but can act themselves. Nanorobots could be controlled by larger IoT systems and, thus, can be used to monitor health or the environment, deliver drugs (for humans) or pheromons (for animals), process waste, develop active clothes or novel filter techniques. Quantum Dot LEDs could be applied for illumination or new user interface designs. The interconnection of several nanoscale devices leads to nanonetworks, and their connection to the internet leads to the Internet of Nanothings (IoNT) - a term invented by Akyildiz and Jornet. In their reference architecture, nano-nodes can communicate with each one over very short distances, nano-routers with more resources to aggregate information and to party control the behavior of the nodes, and nano-micro interfaces that communicate both with the nano devices and with traditional networks. Normal gateways provide for the integration to the internet. Communication in nanonetworks can be nano mechanical, acoustic, electromagnetic, chemical or molecular. However, nanomechanical communication requires direct contact between the devices, which limits its applicability. Chemical or molecular communication usually takes place in fluids, which makes it suitable for in-body applications, which is the idea of the Internet of Bio-Nano-Things. The 
Internet of Nano-Things is the natural extension of the IoT to nano networks. Since they can be applied everywhere, practically every device can be now monitored and/or controlled from the IoT. Still, a lot of research is needed to solve fundamental problems and, thus, the Internet of NanoThings is a long-term goal. However, advances in this field could be applied to other areas before.

\section{HARDWARE}

The arrangement of gadgets or physical devices which are responsive in nature, have the capacity to retrieve information and take after the directions is named as IoT Hardware. The hardware in IoT uses RFID (Radio Frequency Identification) system to receive the information which consists of antenna, transceiver and transponder.

\section{a. Nano-technology and new materials:}

At some point of time, the IoT infrastructure may have a sensor that allows responding and reporting when changes occur in the environment. The Nanoworld is an odd place where things happen a whole lot uniquely in contrast to at the large scale (our ordinary) scale. For example, what you normally consider as the properties of a material are extremely only a normal of the properties of the greater part of its segments. When you get down to the sub-atomic level, the powers that influence singular particles and molecules turn out to be more overwhelming. These are a portion of the reasons why we can't simply recoil down general sensors to the Nano scale and put them on Nano devices to make a system.

Two essential answers for this issue incorporate electromagnetic (EM) and atomic nanosensors. EM nanosensors identify changes in electromagnetic waves, considering quantum impacts, while sub-atomic nanosensors supersede working natural correspondence frameworks to convey a coded message. Both of these techniques require considerably less vitality than their bigger partners; reaped mechanical vitality from nanowire vibrations can control EM nanosensors, and natural biochemicals can be utilized as fuel for atomic nanosensors. Both of these strategies have their individual disadvantages, be that as it may. The significant downside with EM technique is the clamor and diminishment of the EM wave because of retention by encompassing molecules. With the atomic strategy, a major hazard is losing information because of natural impacts, for example, movement or synthetic compounds.

\section{b. Biodegradable antennas:}

Another light-gathering receiving wire complex could prepare for having low-natural effect, organically based sunlight based cells. A biodegradable antenna can be produced using a self-amassing blend of DNA, changed cow-like serum egg whites, and four fluorescent colors. One color ties to the minor section of the DNA twofold helix; the other three tie to particular destinations on the egg whites. The color stacked protein thus ties to the contrarily charged DNA in light of the fact that the analysts synthetically altered the egg whites to be emphatically charged. The course of action permits effective vitality exchange between the colors. With the present arrangement of colors, the receiving wire assimilates blue light and after that radiates for the most part red. The general effectiveness of the radio wire in changing over blue to red photons is just $23 \%$.

\section{c. Autonomous "bee" type devices:}

IoT Enabled Bee Health solution developed using Microservices, IoT, and Cloud architecture. These sensors are manually fitted to bees and work similar to a vehicle etag system; with strategically placed receivers identifying the bee's movement, data is recorded in Data61[2]. Relayr's middleware platform then utilizes an MQTT message broker to send real-time alerts based on the rules engine configuration to collaboration tools Cisco Tropo and Cisco Spark[3]. Relayr has time series data APIs allowing the Cisco Zeus platform to pull data from these APIs for analytics. Also, these time series data APIs are utilized by Micro services to pull the data and load it in a database to integrate, analyze, and visualize.

\section{Data And Signal Processing TECHNOLOGY:}

Digital Signal Processing has ability to receive radio signals used in digital audio components like mp3, medical imaging. Microcontrollers often handle DSP chores and often include DSP instructions. Microcontrollers also have (Analog to Digital Converter) ADCs and (Digital to Analog Converter) DACs on-chip.

\section{Cognitive processing and optimisation}

Cognitive processing of IoT involves understanding the data extracted from sensors, and provide solution for problems and also automation decision from system. Manufacturers have been assembling and securing data for the purposes behind improving undertakings since the essential Industrial Revolution. As data has created in estimate and multifaceted nature, it has turned out to be unquestionably hard to understand the yield. The possibility of a mental methodology lies in the pivotal ability to take existing data and inspect it to drive decisions around improved undertakings, quality, and security. By looking at both sorted out data being accumulated in databases, and unstructured data, for instance, photos or video film, it is possible to pass on more confirmation to essential administration and business exercises.

Optimizing resources, regardless of whether it be individuals, vitality, or information, is basic to minimizing expenses and enhancing general commitment and efficiency levels. Utilizing IoT and cognitive bits of knowledge, it's conceivable to use inputs like geolocation information, use information, singular information, and natural conditions, joined with investigation, to:

- Enhance specialist wellbeing and increase better workforce administration.

- Increment specialist profitability and mastery.

- Lessen vitality utilization of your offices and structures. 


\section{DISCOVERY AND SEARCH ENGINE TECHNOLOGIES:}

IoT data discovery specifies both the activities that are particular to data providers and end publishers. The discovery of the process contains two loops:

- Foraging loop, extract the information, identifies and validates the data that was presented.

- Sense making loop, process the data, analyzes and generate relevant context

\section{a. Cognitive search engines:}

Another novel business seek arrangements that utilize AI advancements, for example, regular dialect handling and machine figuring out how to ingest, comprehend, sort out, and inquiry computerized content from different information sources. Cognitive search engines are distinctive in light of the fact those they Scale to deal with a huge number of information sources and sorts, Utilize man-made reasoning advances, Empower engineers to fabricate seek applications.

\section{b. Autonomous search engines:}

As the number of devices increases, the data that need to be retrieved also increases. To extract the useful data, with the more devices connected to internet a search engine is used. An autonomous search engine should use the technology advance in artificial intelligence and take autonomous decision by evaluating situation devices connected to the network. Autonomous search engines should provide security for the sensory data that is available over the internet and perform the task accordingly.

\section{Power And Energy Storage TECHNOLOGIES:}

Remote sensor systems have turned out to be basic to empowering various applications for the Internet of Things, for example, natural detecting or social insurance observing. Driving these systems has been the focal point of huge research endeavors because of the requirement for dependable and persistent task of the detecting hubs. These necessities are utilized as a system for looking over as far as possible and improvements for different power sources, including energy storage, power distribution, and power rummaging strategies. At long last, we finish up by recognizing that collecting methods are to a great extent deficient for fueling IoT hubs because of constrained power densities or irregularities with reference to when control is gathered; in like manner, coordinate wiring and capacity sources, for example, batteries are the most encouraging methodologies for IoT applications.

\section{a. Biodegradable batteries:}

An adaptable battery made of silk movies could control transitory medicinal sensors and embeds in the body. Utilizing slim silk films for the battery by dissolving the protein fibroin, got from silkworm casings, in water. They spread the arrangement in a shape and peeled off ultrathin sheets of silk after the water dissipated. To make a strong electrolyte for the battery, the specialists mixed a bit of the silk film with the ionic fluid choline nitrate, a liquid salt that behaviors particle[5]. Saving a magnesium compound on another bit of silk shaped an anode, and storing gold on still another piece framed a cathode. The group amassed the battery by sandwiching the electrolyte film between the two cathode films. The postage-stamp-sized, $170-\mu \mathrm{m}-$ thick gadget created a voltage of $0.87 \mathrm{~V}$, which would be sufficient to control an implantable restorative sensor. At the point when put in a saline support arrangement, the gadget about totally decayed following 45 days.

\section{b. Nano-power processing unit:}

$32 \mathrm{~nm}$ or $20 \mathrm{~nm}$ transistor technology is existed already. (e.g. IBM, QUALCOMM, Samsung).World's smallest transistor is based on a graphene nano ribbon just 1 atom x 10 atoms (1 nm transistor) whose Operating frequency close to $1 \mathrm{THz}[1]$. Researchers are working further to reduce the size of these processors for better results.

\section{SECURITY AND PRIVACY TeChNOlogies: Security mechanisms}

The security mechanisms designed to protect communications with the previously discussed protocols must provide appropriate assurances in terms of confidentiality, integrity, authentication and nonrepudiation of the information flows.

a. Anonymity: Anonymity is the concept of decoupling or removing the connection to a particular user from the data collected. As such, no individual user should be identifiable given the data that has been collected. This is a common concern for big data and given the vast quantity of information that IoT devices are expected to generate, it will fall within this field.

b. Digital Forgetting: Digital forgetting is the idea of completely and provably removing an item or piece of data from the digital world. Given the vast amount of data that IoT devices are forecasted to collect, a considerable quantity will probably be sensitive in nature. Therefore, having the ability to be certain that information is deleted after it is deemed no longer necessary is very important.

\section{STANDARDIZATION}

Organizations which are currently working for standardization of IoT protocols [14]:

1. ETSI (European Telecommunications Standards Institute) - Connecting Things Cluster

2. IETF (Internet Engineering Task Force)

- CoRE working group (Constrained RESTful Environments)

- 6 low pan working group (IPv6 over Low power WPAN).

- ROLL working group (Routing Over Low power and Lossy networks).

3. IEEE (Institute of Electrical and Electronics Engineers) - IoT "Innovation Space"

4. OMG (Object Management Group) - Data Distribution Service Portal. 
5. OASIS (Organization for the Advancement of Structured Information Standards) - MQTT Technical Committee.

6. OGC (Open Geospatial Consortium) - Sensor Web for IoT Standards Working Group.

7. IoT-A

"The European Lighthouse Integrated Project representing the Internet-of-Things Architecture, advocates the development of an architectural reference model in conjunction with the definition of an initial set of key building blocks."

8. OneM2M

"The purpose and goal of oneM2M is to develop technical specifications which address the need for a common M2M Service Layer that can be readily embedded within various hardware and software, and relied upon to connect the myriad of devices in the field with M2M application servers worldwide."

\section{OSIOT}

"It is an organization with a single focus to develop and promote royalty-free open source standards for the emerging Internet of Things."

10. IoT-GSI (Global Standards Initiative on Internet of Things)

11. ISA International Society of Automation

12. W3C

- Semantic Sensor Net Ontology.

- Web of Things Community Group.

\section{CONCLUSION}

Our paper derives a sincere attempt in presenting a research roadmap for IoT practitioners who want to delve into this popular technology for next 10 years and beyond. They should be able to construct a vision with confidence which is possible only by knowing the factors which influence the performance of an IoT system. In this paper we have discussed about diverse aspects which will affect the design of an IoT system in years to come. We present a cautious conclusion by advocating highly specialized technological mechanisms to maximize the throughput of the IoT system thereby neutralizing the need of technologies which may be obsolete beyond 2025 .

\section{REFERENCES}

[1].https://www.brookings.edu/blog/techtank/2015/09/29/n anotechnology-promises-powerful-new-applications-forthe-internet-of-things/

[2].https://dzone.com/articles/connected-bees-iot-enabledbee-health

[3].https://cen.acs.org/articles/95/i16/Makingbiodegradable-batteries-silk.html

[4].https://medium.com/@ maheshwar.ligade/architecturefor-IoT-applications-d50ece $031 \mathrm{~d} 38$

[5].https://cen.acs.org/articles/93/i33/DNA-Protein-Self-

Assemble-Biodegradable.html

[6].https://ndiastorage.blob.core.usgovcloudapi.net/ndia/20

16/armament/18399_Tillinghast.pdf

[7].Routledge, The Internet of Things: From RFID to the NextGeneration Pervasive Networked Systems (Hardback) - Routledge,(2008).
[8].M. Zorzi, A. Gluhak, S. Lange, A. Bassi, From Today's Intranet of Things to a Future Internet of Things: A Wireless- and Mobility Related View, IEEE Wirel Commun. 17 (2010) 43-51.

[9].N. Honle, U.P. Kappeler, D. Nicklas, T. Schwarz, M. Grossmann, Benefits of Integrating Meta Data into a Context Model, in: 2005: pp. 25-29.

[10].A. Gluhak, S. Krco, M. Nati, D. Pfisterer, N. Mitton, T. Razafindralambo, A Survey on Facilities for Experimental Internet of Things Research, IEEE Commun Mag. 49 (2011) 58-67.

[11].L. Haiyan, C. Song, W. Dalei, N. Stergiou, S. KaChun, A remote markerless human gait tracking for ehealthcare based on contentaware wireless multimedia communications, IEEE Wirel Commun.(2010) 44-50.

[12].G. Nussbaum, People with disabilities: assistive homes and environments, Computers Helping People with Special Needs. (2006).

[13].A. Alkar, U. Buhur, An Internet based wireless home automation system for multifunctional devices, IEEE $\mathrm{T}$ Consum Electr. 51 (2005) 1169-1174.

[14].M. Darianian, M.P. Michael, Smart Home Mobile RFID-based Internet-Of-Things Systems and Services, 2008 International Conference on Advanced Computer Theory and Engineering. (2008) 116-120.

[15].International Data Corporation (IDC), Explosive Internet of Things Spending to Reach $\$ 1.7$ Trillion in 2020, According to IDC, 02 June 2015, [Online]. Available: http://www.idc.com/getdoc.

jsp?containerId=prUS25658015. [Accessed 22 August 2016].

[16].Gartner Inc., The Internet of Things Is a Revolution Waiting to Happen, 30 April 2015. [Online]. Available: http://www.gartner.com/smarterwithgartner/the-internet-

of-things-is-a-revolution-waitingto-happen. [Accessed 22 August 2016].

[17].IoT-Based Big Data: From Smart City towards Next Generation Super City Planning ,M. Mazhar Rathore,et.al.,International Journal on Semantic Web and Information Systems, Volume 13 - Issue 1, January-March 2017.

[18].S. E. Khateeb, "IoT architecture a gateway for smart cities in Arab world," 2018 15th Learning and Technology Conference (L\&T), Jeddah, 2018, pp. 153-160.doi: 10.1109/LT.2018.8368500.

[19].Ghajargar, M., Wiberg, M., \& Stolterman, E. (2018). Designing IoT systems that support reflective thinking: A relational approach. International Journal of Design,

12(1), 21-35.

[20].Janlert, L.-E., \& Stolterman, E. (2017). The things that keep us busy: The elements of interaction. Cambridge, MA: MIT Press. 